\title{
Motor learning in childhood reveals distinct mechanisms for memory retention and re-learning
}

\author{
Kristin E. Musselman, ${ }^{1,2}$ Ryan T. Roemmich, ${ }^{1,2}$ Ben Garrett,, ${ }^{1,2}$ and Amy J. Bastian ${ }^{1,2}$ \\ ${ }^{1}$ The Solomon H. Snyder Department of Neuroscience, The Johns Hopkins University School of Medicine, Baltimore Maryland 21205, \\ USA; ${ }^{2}$ Motion Analysis Laboratory, The Kennedy Krieger Institute, Baltimore, Maryland 21205, USA
}

\begin{abstract}
Adults can easily learn and access multiple versions of the same motor skill adapted for different conditions (e.g., walking in water, sand, snow). Following even a single session of adaptation, adults exhibit clear day-to-day retention and faster re-learning of the adapted pattern. Here, we studied the retention and re-learning of an adapted walking pattern in children aged 6-17 yr. We found that all children, regardless of age, showed adult-like patterns of retention of the adapted walking pattern. In contrast, children under 12 yr of age did not re-learn faster on the next day after washout had occurred-they behaved as if they had never adapted their walking before. Re-learning could be improved in younger children when the adaptation time on day 1 was increased to allow more practice at the plateau of the adapted pattern, but never to adult-like levels. These results show that the ability to store a separate, adapted version of the same general motor pattern does not fully develop until adolescence, and furthermore, that the mechanisms underlying the retention and rapid re-learning of adapted motor patterns are distinct.
\end{abstract}

Learning new motor skills is an ongoing task during childhood. Not only must children learn fundamental motor skills, such as walking, they must learn to adapt these skills in response to changes in the environment (e.g., an icy walking surface) and changes within themselves (e.g., walking with a sprained ankle). This type of learning, whereby an established motor pattern is modified in response to a predictable perturbation to the movement, is called motor adaptation (Martin et al. 1996). Motor adaptation is driven by errors in the movement and requires an intact cerebellum (Morton and Bastian 2006).

Adapted motor patterns can be stored and recalled, enabling previous experience to facilitate faster re-learning (Medina et al. 2001; Huang et al. 2011; Malone et al. 2011). Healthy adults show smaller movement errors and faster adaptation when presented with a familiar perturbation (called "retention") (Joiner and Smith 2008; Malone et al. 2011). Furthermore, adults show this faster re-learning even if the adapted pattern has been "washed-out" or unlearned, demonstrating memory of a different version of the same motor skill (Huang et al. 2011; Malone et al. 2011). This faster re-learning is important not only for successful movement in everyday life, but it can also facilitate motor learning in rehabilitation (Reisman et al. 2013).

Compared with adults, children acquire adapted motor patterns more slowly over a single training session, but can achieve a similar magnitude of learning (Jansen-Osmann et al. 2002; Konczak et al. 2003; Musselman et al. 2011; Vasudevan et al. 2011; Patrick et al. 2014). This difference likely reflects the immaturity of the nervous system, especially the cerebellum (Morton and Bastian 2006; Vasudevan et al. 2011), which continues to develop for at least the first decade of life (Caviness et al. 1996; Saksena et al. 2008; Tiemeier et al. 2010). Do children and adults also differ in the retention and re-learning of adapted motor patterns? To study motor adaptation in children aged 6-17 yr, we used a split-belt treadmill that has separate belts for each leg, allowing one belt to move faster than the other (Musselman et al. 2011; Vasudevan et al. 2011). Split-belt walking creates temporal

\section{Corresponding author: bastian@kennedykrieger.org}

Article is online at http://www.learnmem.org/cgi/doi/10.1101//m.041004. 115. and spatial asymmetries in one's gait (Reisman et al. 2005), necessitating adaptation of the gait pattern. More specifically, it creates asymmetries in step length, spatial coordination (measured by the point of oscillation of each leg, called the center of oscillation), and temporal coordination (measured by the phasing between the legs). We hypothesized that, like the acquisition of an adapted motor pattern, the abilities to retain and re-learn would be attenuated in children compared with adults. Furthermore, we hypothesized that retention and re-learning would emerge at the same age since they are thought to involve the same neural structures and mechanisms (Medina et al. 2001). Contrary to our expectations, we show that while children of all ages retained the adapted gait pattern, children under age 12 were unable to utilize previously adapted gait patterns to facilitate rapid re-learning.

\section{Results}

We studied 71 healthy children aged 6-17 yr who were divided into two groups (Retention and Re-learning), and each performed two testing sessions spaced 24-h apart (Fig. 1). On day 1 both groups began by walking with the treadmill belts moving at the same speed (called "tied belts") and then walked with the belts split for $15 \mathrm{~min}$. On day 2, the Retention group immediately walked in the same split-belt condition experienced on day 1 . This allowed us to assess the children's ability to retain learning over a 24-h period. On day 2, the Re-learning group first washed out the adapted motor pattern by walking on tied belts for 15 min. This group then readapted to the same split-belt condition experienced on day 1 . This allowed us to assess the children's ability to reaccess a previously learned motor skill after it had been washed out. Two example participants from the 12 to 14 -yr-old age group are shown in Figure 1 for each group. Note that these subjects showed minimal step length asymmetry during day 2

(C) 2016 Musselman et al. This article is distributed exclusively by Cold Spring Harbor Laboratory Press for the first 12 months after the full-issue publication date (see http://learnmem.cshlp.org/site/misc/terms.xhtml). After 12 months, it is available under a Creative Commons License (AttributionNonCommercial 4.0 International), as described at http://creativecommons. org/licenses/by-nc/4.0/. 
A Retention

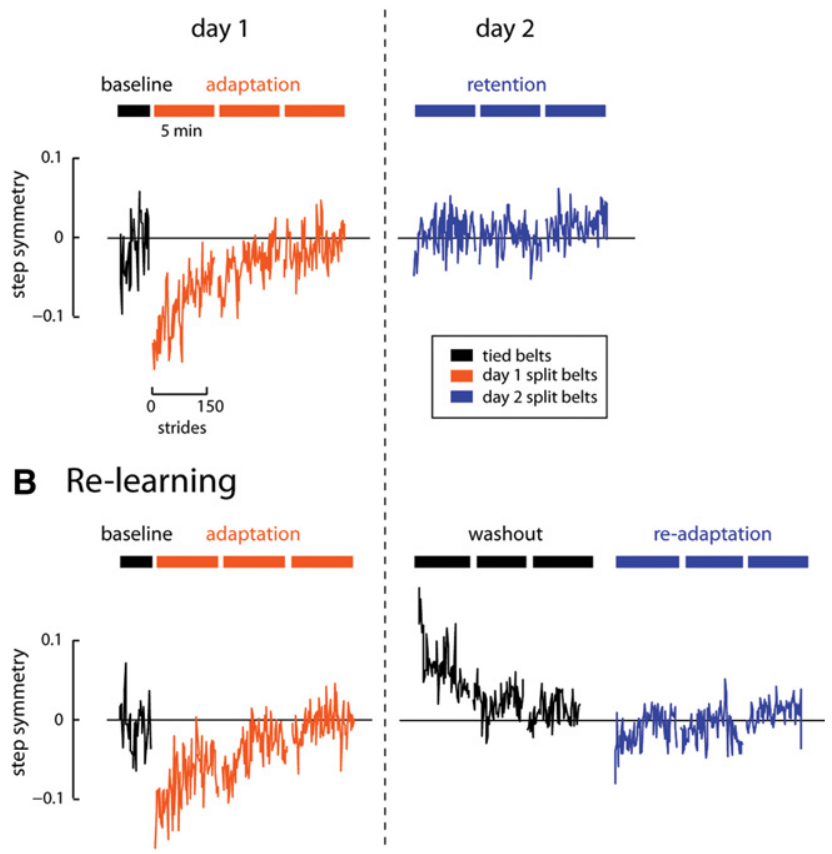

Figure 1. Schematic of paradigm and results from example subjects in the 12- to 14-yr-old age group for $(A)$. The Retention group and $(B)$. The Re-learning group. Step symmetry is plotted for consecutive strides. Note that day 2 performance is improved in these adolescents, regardless of whether learning had been washed out or not.

split-belt walking. Importantly, the washout experienced by the Re-learning participant did not eliminate the adapted pattern learned on day 1.

All of the children completed the experiments on days 1 and 2. Data were excluded from four children for the following reasons: (1) Child looked down at his/her feet despite being asked not to and appeared to be consciously changing his/her walking pattern, causing exaggerated and deliberate stepping (two children, 9 and $17 \mathrm{yr}$ old). Such conscious strategies are known to affect split-belt adaptation (Malone and Bastian 2010). (2) Child's initial gait asymmetry on day 1 was approximately two times greater than that of the other children (1 child, 12 yr old). (3) Child's aftereffect on day 2 did not wash out with tied-belt walking ( 1 child, 15 yr old). Of the remaining 67 children, all showed adaptation in step length symmetry on day $1(P<0.05$ for independent $t$-test comparing step length symmetry values during early [i.e., first 20 steps with belts split] and late [i.e., last 20 steps with belts split] adaptation for each child). Thus, learning occurred and all 67 children (36 males) were included in the analysis. Age group (number of children) was as follows: 6-8 yr old (21), 9-11 yr old (15), 12-14 yr old (16), 15-17 yr old (15).

Before comparing day-to-day patterns of retention and re-learning, we show that day 1 measures at baseline, early adaptation (reflecting perturbation size) and late adaptation (reflecting the amount that was learned) were similar across the experimental groups and age groups. Table 1 shows the statistical results for step symmetry, the center of os-

Table 1. Day 1 statistics cillation symmetry, and phase. This demonstrates that children of all ages in both the Retention and Re-learning groups experienced the same amount of error and achieved a similar adapted gait pattern by the end of day 1 . This was the case despite the fact that the younger children tended to adapt at a slower rate than the older children, consistent with our prior study (Vasudevan et al. 2011).

We then assessed whether, in the Re-learning group, children in different age groups showed similar aftereffects and washout on day 2. This was only done for the Re-learning group since the Retention group did not undergo washout. Children in all age groups showed similar step symmetry aftereffects during early washout ( $F=1.85, P=0.16$, Fig. 2$)$. This was also true for the center of oscillation symmetry $(F=1.77, P=0.18)$ and phasing $(F=1.82, P=0.17)$. Children in all age groups showed similar washout of step symmetry after 15 min of tied-belt walking (Fig. 2C; open dots on right plots Fig. 3B). There were no differences in the symmetry values of the last 20 steps of the washout period across ages for step length $(F=0.23, P=0.87)$, center of oscillation $(F=1.54, P=0.23)$, or phasing $(F=0.72, P=0.55)$.

\section{Step length symmetry retention and re-learning}

All children in the Retention group showed a smaller initial perturbation in step length during the early adaptation period on day 2 (compare orange and blue adaptation traces in Fig. 3A). This finding was seen across all age groups in the Retention group, suggesting that children as young as $6 \mathrm{yr}$ of age show retention of an adapted gait pattern. In contrast, not all children in the Relearning group showed a smaller initial perturbation on day 2 compared with day 1 (see Fig. 3B). The orange and blue adaptation traces overlap for the $6-8$ and $9-11$ yr olds, suggesting no rapid relearning of the adapted gait pattern on day 2. Only the 12-14 and 15-17 yr olds show rapid re-learning after washout on day 2 (i.e., less initial perturbation on day 2).

Early adaptation difference for each age and experiment group is shown in Fig. 3C,D. A two-way ANOVA (age group $\times$ experimental group) showed significant main effects of experimental group $(F=51.46, P<0.001)$ and age $(F=5.23, P=0.003)$. This suggests that the motor memory remaining on day 2 was greater for the Retention group, and greater in older children, respectively. The interaction (age group $\times$ experimental group) was also statistically significant $(F=3.45, P=0.023)$. Within the Re-learning group, post hoc comparisons revealed significant differences $(P \leq 0.0016)$ in early adaptation difference between the 15-17 yr olds and the two younger age ranges (see Fig. 3D).

We used Pearson's correlation to understand if these relationships held when looking across individual subjects in the Retention and Re-learning group. Figure 4A shows that, in the Retention group, the early adaptation difference from day 1 to

\begin{tabular}{|c|c|c|}
\hline Step symmetry & Center of oscillation & Phase \\
\hline $\begin{array}{l}\text { Baseline } \\
\text { Group: } F=0.01, P=0.90 \\
\text { Age: } F=0.26, P=0.85 \\
\text { Interaction: } F=0.08, P=0.97\end{array}$ & $\begin{array}{l}\text { Group: } F=1.47, P=0.23 \\
\text { Age: } F=1.40, P=0.25 \\
\text { Interaction: } F=0.32, P=0.81\end{array}$ & $\begin{array}{l}\text { Group: } F=1.83, P=0.18 \\
\text { Age: } F=1.69, P=0.18 \\
\text { Interaction: } F=0.35, P=0.79\end{array}$ \\
\hline \multicolumn{3}{|l|}{ Early adapt (perturbation) } \\
\hline $\begin{array}{l}\text { Group: } F=0.16, P=0.69 \\
\text { Age: } F=0.44, P=0.72 \\
\text { Interaction: } F=0.42, P=0.74\end{array}$ & $\begin{array}{l}\text { Group: } F=0.00, P=0.96 \\
\text { Age: } F=0.36, P=0.78 \\
\text { Interaction: } F=0.78, P=0.51\end{array}$ & $\begin{array}{l}\text { Group: } F=0.39, P=0.53 \\
\text { Age: } F=0.07, P=0.98 \\
\text { Interaction: } F=0.50, P=0.68\end{array}$ \\
\hline \multicolumn{3}{|l|}{ Late Adapt } \\
\hline $\begin{array}{l}\text { Group: } F=1.13, P=0.29 \\
\text { Age: } F=1.90, P=0.14 \\
\text { Interaction: } F=0.59, P=0.62\end{array}$ & $\begin{array}{l}\text { Group: } F=0.02, P=0.88 \\
\text { Age: } F=0.85, P=0.47 \\
\text { Interaction: } F=0.61, P=0.61\end{array}$ & $\begin{array}{l}\text { Group: } F=0.18, P=0.67 \\
\text { Age: } F=0.24, P=0.87 \\
\text { Interaction: } F=0.22, P=0.88\end{array}$ \\
\hline
\end{tabular}



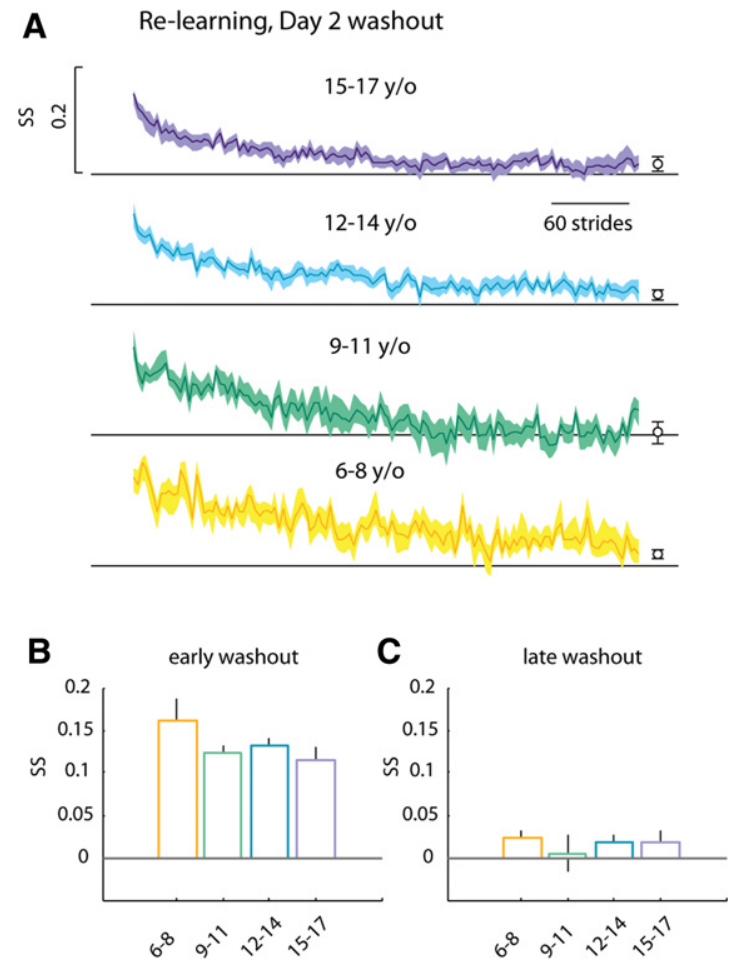

Figure 2. (A) Step symmetry (SS) for the first 390 strides during the washout for the Re-learning group on day 2. (B) Mean step symmetry for early washout (first 20 strides). There were no significant differences in early washout, though the 6- to 8-yr-old group showed a slightly larger aftereffect. (C) Mean step symmetry for late washout (last 20 strides). There were no significant differences in late washout.

day 2 was not correlated with age $(r=0.08, P=0.65)$. In contrast, Figure $4 \mathrm{~B}$ shows that there was a moderately strong correlation between age and the amount of change from day 1 to day 2 in the Re-learning group $(r=0.62, P<0.001)$. Younger children were unable to benefit from learning the prior day. This was not simply due to the fact that younger children had more variable baseline walking patterns. Stepwise regression was used to determine whether age and variance of baseline step length symmetry contributed to predicting the early adaptation difference from day 1 to day 2 . Age was the only regressor that was included in the model $\left(R^{2}=0.37, F=16.45, P<0.001\right)$ - the variance term did not add to the prediction, nor did it predict the result as well as age.

\section{Adaptation of center of oscillation and phasing across days} The center of oscillation represents a spatial parameter that contributes to changes in step symmetry during learning. Figure 5A shows that children in the Retention group showed a smaller initial perturbation in center of oscillation during the early adaptation period on day 2, though this effect was reduced for the 6-8 yr olds. Like step length symmetry, the younger children in the Re-learning group (i.e., ages $6-11$ yr) did not show rapid re-learning of the adapted gait pattern on day 2 (orange and blue traces overlap in the top two plots in Fig. 5B), whereas the oldest two age groups (12-14 and 15-17 yr old) did.

A two-way ANOVA (age group $\times$ experimental group) was run on the early adaptation difference data for the center of oscillation. A significant main effect was found for experimental group $(F=5.27, P=0.026)$. The main effect of age and the interaction were not significant $(F=0.36, P=0.79$ and $F=1.43, P=0.25$, respectively). Thus, with respect to center of oscillation, the motor memory of the adapted gait remained on day 2 for the Retention group, but not the Re-learning group.

Children of all ages showed retention in phasing, which is a measure in the temporal domain (Fig. 6A). No rapid re-learning of the adapted gait pattern on day 2 was seen in the children aged 6$8 \mathrm{yr}$ (Fig. 6B). In contrast to the results for step length and center of oscillation, the 9-11 yr olds did show rapid re-learning in phasing that was comparable with the older age groups.

When the early adaptation difference for phasing was compared across age groups and experimental groups (Fig. 6C,D; 2-way ANOVA), significant main effects were found for experimental group $(F=21.54, P<0.001)$, but the main effect of age and the interaction were not significant $(F=0.41, P=0.75$ and $F=1.63, P=0.19$, respectively).

\section{Effects of extended training on day 1 in 6-8 year olds}

Since children $<12$ yr old in the Re-learning group did not show faster re-learning on day 2 , we wondered whether increasing the exposure to split-belt walking on day 1 would facilitate faster relearning on day 2 . To assess this, an additional group of children aged 6-8 yr were tested (called "Extended" group). Seven children (3 males) aged $6-8 \mathrm{yr}$ participated in extended training on day 1 (i.e., $30 \mathrm{~min}$ of split-belt walking). Their data were compared with that of the $6-8$ yr old Re-learning group ( $n=7,4$ males). There were no differences in day 1 baseline behavior between the two groups (baseline symmetry compared for step length $(P=0.18)$, center of oscillation $(P=0.22)$, phase $(P=0.82))$. Likewise, there were also no differences in day 1 adaptation behavior between the Re-learning and Extended groups. The size of the initial perturbation (i.e., early adaptation) was the same for step length symmetry $(P=0.32)$, center of oscillation symmetry $(P=0.75)$ and phasing $(P=0.27)$. The late adaptation period, which reflects the level of symmetry where adaptation plateaued, also did not differ between the two groups (step length symmetry $(P=0.86)$, center of oscillation symmetry $(P=0.91)$, phase $(P=$ 0.91)). On day 2, the children in the Re-learning and Extended groups showed a similar aftereffect in step length symmetry, center of oscillation symmetry, and phasing during tied-belt walking ( $P=0.81$ for step length, $P=0.35$ for center of oscillation, $P=0.64$ for phase). They also washed out the aftereffect, or learning, to a similar extent on day 2 . There were no differences in the symmetry values of the last 20 steps of the washout period ( $P=0.26$ for step length, $P=0.22$ for center of oscillation, $P=$ 0.08 for phase).

Since the two groups were similar in baseline, adaptation and washout behavior, any differences seen in the re-learning on day 2 can be attributed to the extended training on day 1 . On day 2 the extended group showed more rapid re-learning of step length and center of oscillation symmetry, but not phasing, compared with day 1 (step length symmetry data in Fig. 7). The early adaptation difference was compared across the three groups of 6-8 yr olds (i.e., Retention, Re-learning, and Extended). For step length symmetry, the difference in perturbation across days differed significantly across groups $(F=20.7, P<0.0001$, one-way ANOVA). Post hoc analyses showed the early adaptation difference to be significantly greater (indicating greater retention/re-learning) in the Retention and Extended groups relative to the Re-learning group $(P<0.05)$. For center of oscillation symmetry, the results were similar to those for step length symmetry, however, there were no significant differences in early adaptation difference between the three groups ( $F=1.34, P=0.29$, one-way ANOVA). Significant differences in early adaptation difference were seen for phasing ( $F=12.5, P<0.001$, one-way ANOVA). For this parameter, early adaptation difference was significantly greater in the Retention group, while the Re-learning and Extended groups were similar. 


\section{step symmetry}

A retention

B

re-learning

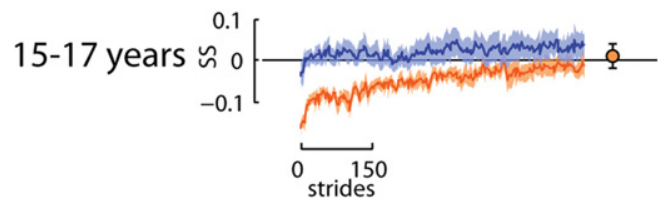

12-14 years

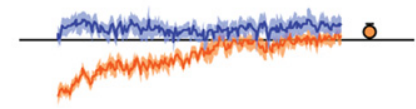

9-11 years

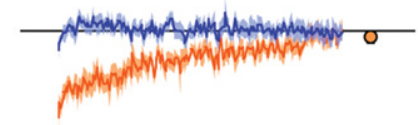

6-8 years

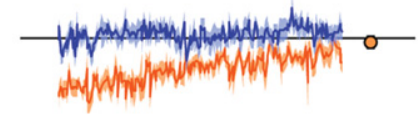

C

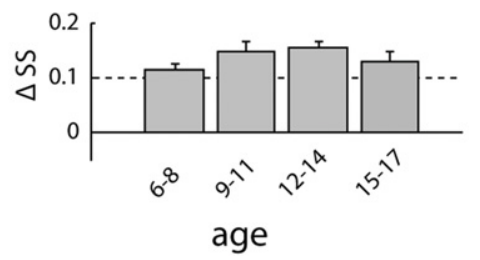

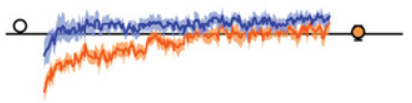
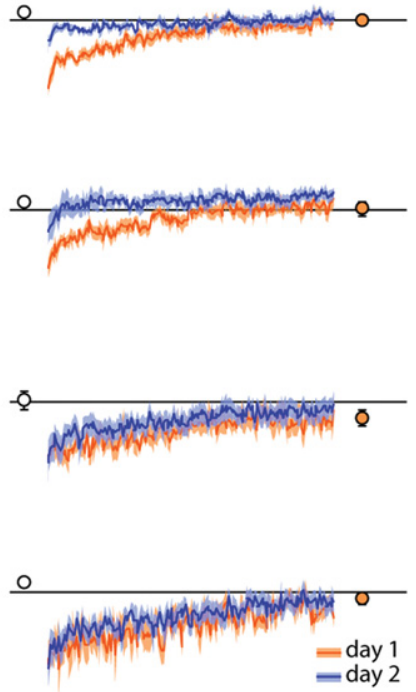

D

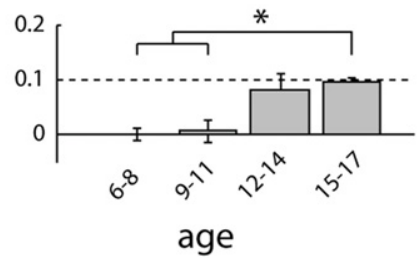

Figure 3. (A) Retention group: step symmetry (SS) across 600 strides of the split-belt conditions on day 1 (orange) and day 2 (blue) overlaid. Solid lines with shaded area are mean \pm SE. Closed symbols show final (last 20 strides) of step symmetry for day 1 learning. (B) Re-learning group data plotted as in $A$. Open symbols show step symmetry prior to re-learning (last 20 strides of washout). Note that the curves overlap for the two youngest groups, indicating no improvement in re-learning. (C) Difference across days (day 2-day 1 ) in step symmetry during early adaptation (first 20 strides of split-belt walking) for the Retention group. (D) Difference in step symmetry for the Re-learning group plotted as in C. The two youngest age groups were significantly different from the oldest age group (see main text).

\section{Discussion}

Children are thought to have superior learning abilities compared with adults across many domains, including the acquisition of motor skills. Surprisingly, this study shows that the critical ability to store a separate adapted version of the same general motor pattern for use during re-learning does not fully develop until adolescence. Instead, the memory of a recently adapted motor state is reversible in children up to around $12 \mathrm{yr}$ of age. This was not due to a deficit in retaining motor memories since children as young as $6 \mathrm{yr}$ of age could retain an adapted pattern from one day to the next as long as no washout occurred. This suggests that the children erase the motor memory during washout, whereas adolescents may switch to a different stored pattern, and then switch back when needed (Kitago et al. 2013). This result is consistent with the observation that children have difficulty switching between motor patterns for varying demands of a given task (Forssberg et al. 1995; Dominici et al. 2010). The findings also suggest that the mechanisms underlying the retention and rapid re-learning of adapted motor patterns must be distinct. Lastly, we found that increasing the duration of training, or exposure

to split-belt walking, on day 1 could partially improve re-learning of spatial parameters on day 2 . This suggests that reinforcement of the adapted motor pattern is one ingredient for improving re-learning.

\section{Learning rates and re-learning}

The rate of motor adaptation in children differs notably from that seen in adults for both reaching (Jansen-Osmann et al. 2002; Konczak et al. 2003) and walking adaptation (Musselman et al. 2011; Vasudevan et al. 2011). Adaptation in children tends to be slower and its time course more linear compared with adults. In the case of split-belt treadmill walking, this is especially true for the spatial parameters (Musselman et al. 2011; Vasudevan et al. 2011). Not only do children adapt more slowly than adults, they are also missing the initial, fast adaptation seen in adults (Musselman et al. 2011; Vasudevan et al. 2011). Motor adaptation is believed to consist of two learning states: (1) a fast process that very quickly reduces motor error, but contributes little to retention of the adapted state, and (2) a slow process that is slower to respond to the movement error, but can show retention of the adapted pattern upon subsequent exposures (Smith et al. 2006). The slow process has been hypothesized to explain improved re-learning when it is not completely washed out (Smith et al. 2006). However, the two-state adaptation model cannot explain savings after a prolonged washout (Zarahn et al. 2008), as we included in our re-learning experiments here.

We have recently explored the factors that drive the nervous system's ability to accelerate re-learning in adult walking (Roemmich and Bastian 2015). Our work shows that faster re-learning is not due to a single factor-it depends on repetition of the newly learned walking
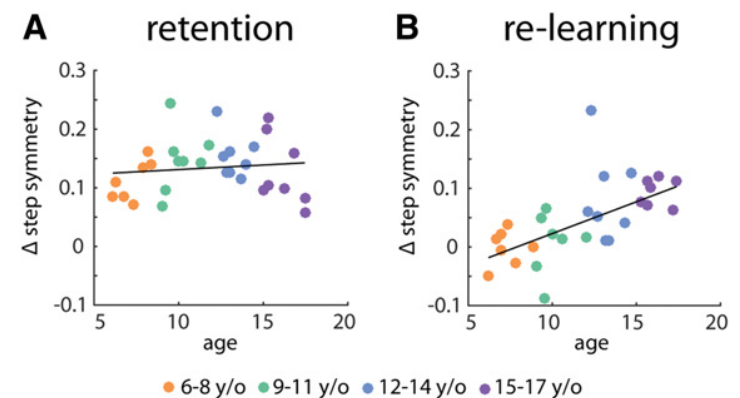

Figure 4. Scatter plot showing relationship between age and difference across days (day 2 - day 1 ) in step symmetry during early adaptation (first 20 strides of split-belt walking) for $(A)$ Retention group and (B) Re-learning group. Age groups coded by color. Lines of best fit included on plots. 


\section{center of oscillation}

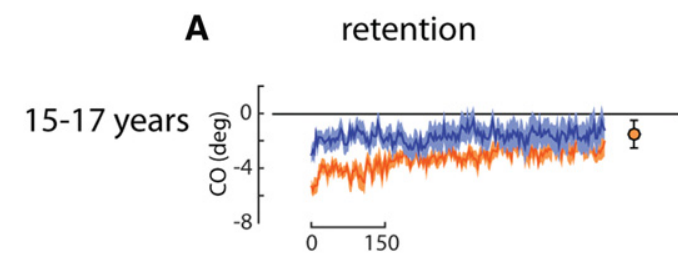

\section{B}

re-learning

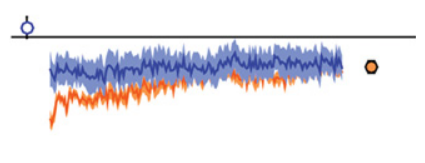

$12-14$ years
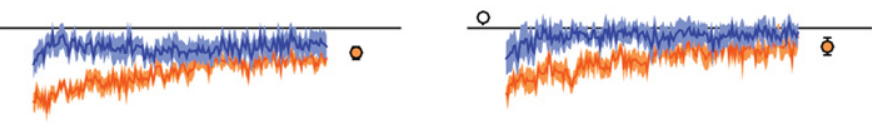

9-11 years
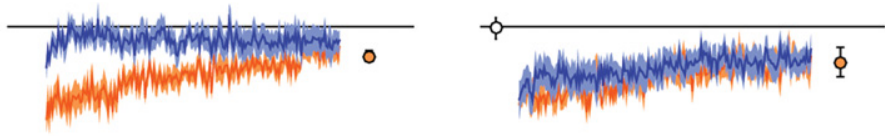

6-8 years

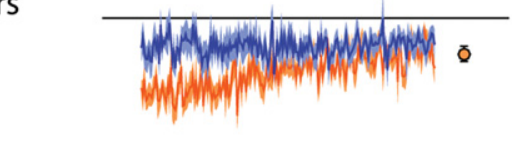

C

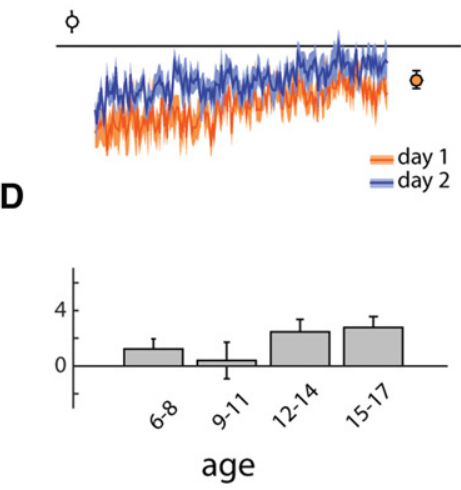

Figure 5. (A) Retention group: symmetry in center of oscillation (CO) across 600 strides of the splitbelt conditions on day 1 (orange) and day 2 (blue) overlaid. Solid lines with shaded area are mean \pm SE. Closed symbols show final (last 20 strides) center of oscillation symmetry for day 1 learning. (B) Relearning group data plotted as in $A$. Open symbols show center of oscillation symmetry prior to re-learning (last 20 strides of washout). (C) Difference across days (day 2-day 1 ) in center of oscillation symmetry during early adaptation (first 20 strides of split-belt walking) for the Retention group. (D) Difference in symmetry for the Re-learning group plotted as in $C$.

pattern in the adapted state (i.e., prolonged adaptation) and the ability to explicitly identify and recall the initial perturbation. Both of these factors improve re-learning and they are additive. When one factor is present without the other, re-learning is only partially improved. These findings demonstrated that at least two distinct neural mechanisms-one that identifies and recalls the learning environment and one that stores and recalls learned walking patterns-contribute to accelerate re-learning. We think that the absence of savings observed in the younger children might be explained by the underdevelopment of one or both of these mechanisms.

We provided further insight into these mechanisms in our second experiment, which showed that young children are able to partially save the learned walking pattern after prolonged practice during initial learning. This finding fits within the framework we put forth to explain savings in adults, and with other ideas about savings of motor learning. For example, other investigators refer to this type of approach as "over-learning" and found that it is related to long-term memory of adapted movements (Joiner and Smith 2008) and operant reinforcement (Huang et al. 2011). We therefore think that children even as young as 6 yr old can store and recall learned walking patterns after an extended learning pe- riod, but savings remains impaired because they likely lack the ability to identify and recall the perturbation.

It is notable that the effect of extended training was different for the temporal measure of phasing. Extended training on day 1 did not facilitate faster re-learning of phasing on day 2. Previous work has also found a dissociation between the spatial and temporal components of split-belt walking adaptation in adults (Malone et al. 2012) and children (Musselman et al. 2011; Vasudevan et al. 2011; Patrick et al. 2014), suggesting different neural substrates and/or processes for spatial and temporal adaptation. Clearly the process responsible for spatial adaptation is responsive to over-training whereas the process for temporal adaptation is not.

\section{Could variable stepping behavior affect retention and re-learning?}

Children, especially young ones, show greater variability in their motor performance compared with adults (Adolph et al. 2015). It is possible that variability in motor behavior could affect learning, retention and re-learning by disrupting the internalization of the motor memory. However, our results indicate that age is the better predictor of re-learning than variability in the motor pattern. This is consistent with previous work from our group (Vasudevan et al. 2011) and others (Takahashi et al. 2003), which showed that increased movement variability did not limit motor adaptation (i.e., learning). Furthermore, in adults increased motor variability during baseline was found to facilitate same-day learning during reaching adaptation (Wu et al. 2014). Our present findings suggest that variability does not interfere with the internalization of motor memory as all children, regardless of age, showed similar retention of the learned motor behavior on day 2 . Thus, we think it unlikely that the variable stepping behavior seen in younger children explains the differences in re-learning across ages.

\section{Protracted brain development may explain attenuated re-learning in young children}

Children younger than $12 \mathrm{yr}$ of age did not show rapid re-learning when exposed to split-belt walking a second time. This is in contrast to the faster rate of re-learning seen in the adolescents in this study, and in healthy adults (Malone et al. 2011). Structural maturation of the sensorimotor nervous system is ongoing for at least the first decade of life (Caviness et al. 1996; Giedd et al. 1999; Gogtay et al. 2004; Østby et al. 2009; Tiemeirer et al. 2010), and this immaturity likely underlies the differences in motor adaptation across ages seen in this study and in others (Vasudevan et al. 2011; Patrick et al. 2014).

Several brain regions may play a role in faster re-learning of adapted motor patterns. The cerebellum has been implicated in behavioral (Morton and Bastian 2006) and in vivo animal studies 


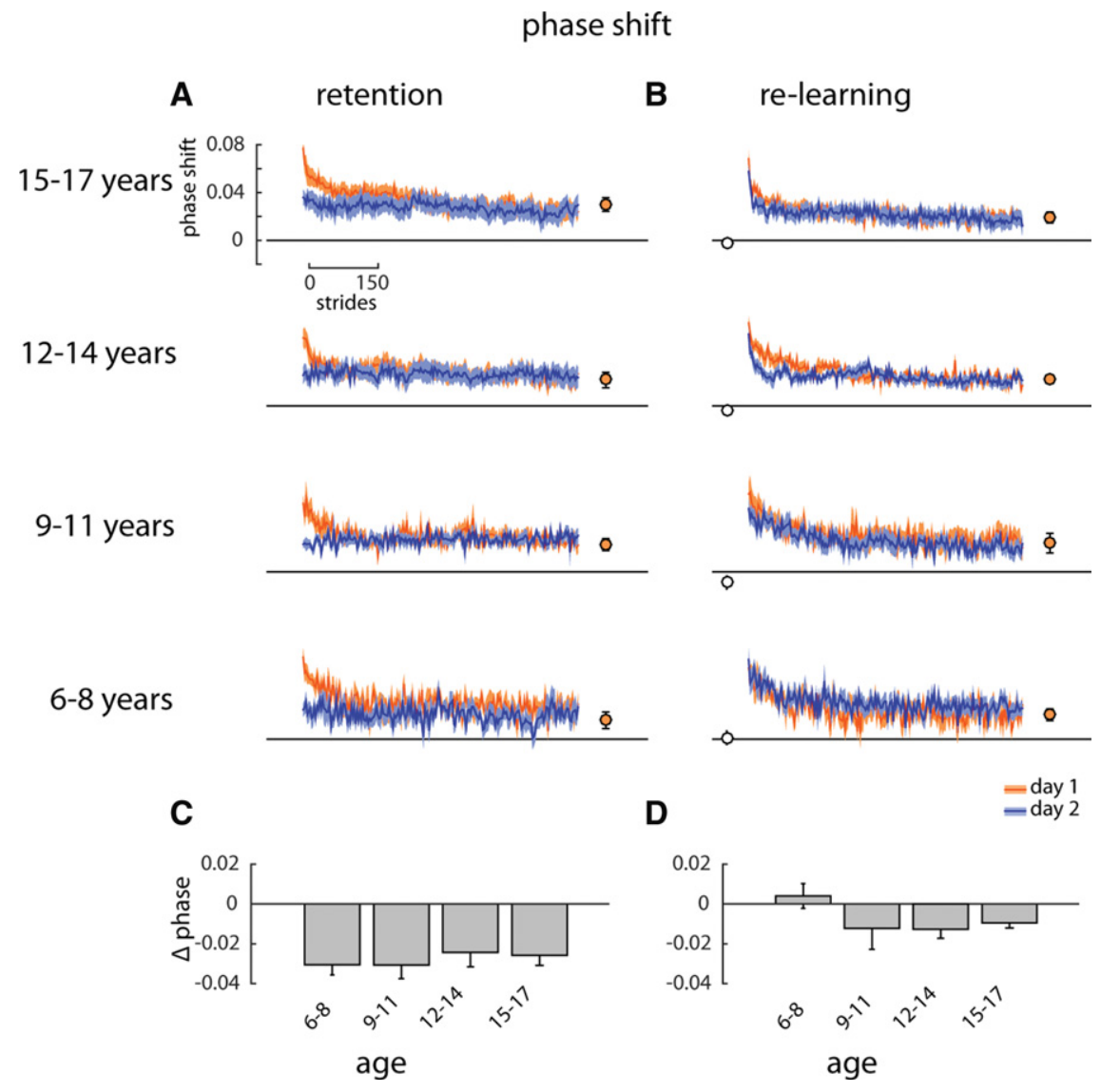

Figure 6. (A) Retention group: phasing across 600 strides of the split-belt conditions on day 1 (orange) and day 2 (blue) overlaid. Solid lines with shaded area are mean \pm SE. Closed symbols show final (last 20 strides) phasing for day 1 learning. (B) Re-learning group data plotted as in $A$. Open symbols show phasing prior to re-learning (last 20 strides of washout). (C) Difference across days (day 2-day 1 ) in phasing during early adaptation (first 20 strides of split-belt walking) for the Retention group. $(D)$ Difference in symmetry for the Re-learning group plotted as in $C$. show protracted development during childhood (Østby et al. 2009), and there is evidence that the basal ganglia plays a role in motor adaptation and the formation of longer-term motor memories (Marinelli et al. 2009; Bédard and Sanes 2011). In individuals with Parkinson's disease, re-learning (savings) of an upper extremity movement is attenuated (Marinelli et al. 2009; Bédard and Sanes 2011), similar to the young children in this study. However, savings of split-belt walking was recently found to be intact in Parkinson's disease (Roemmich et al. 2014). New studies are needed to understand which brain networks are most important for development of mature re-learning patterns.

\section{Clinical implications}

The results of this study may have clinical implications. In general, the younger a child is when a brain injury is experienced, the greater the recovery of mobility (Fritz et al. 2011). The reason for this is unclear, though it is often attributed to heightened "plasticity" during development. While our data represent only one type of motor learning, it is interesting that children learn slower and do not readily benefit when re-learning the same pattern again the next day. Thus, children may require more practice than adults to change their motor pattern, but may have the capacity to improve to a greater extent in the long run. Future work should also investigate how activity between learning sessions
(Medina et al. 2001). Studies of Pavlovian eyeblink conditioning with extinction training in rabbits have shed light on the importance of cerebellar structures for rapid re-learning, also known as savings (Medina et al. 2001). Acquisition of the conditioned response (i.e., eye blink) is associated with plasticity in the cerebellar cortex and the interpositus nucleus; however, only the plasticity in the nucleus persists following extinction training, suggesting it is an important site of savings (Medina et al. 2001). In children, the neural structures and/or functionality required for rapid re-learning, which may include the deep cerebellar nuclei and their connections with the cerebellar cortex, are likely not online until the second decade of life. This timeline corresponds with the protracted maturation of the cerebellum-whole cerebellar volume does not peak until the early teenage years (Caviness et al. 1996; Tiemeier et al. 2010). Likewise, the caudate, putamen and pallidum all

\section{A re-learning in 6-8 yo after extended training}
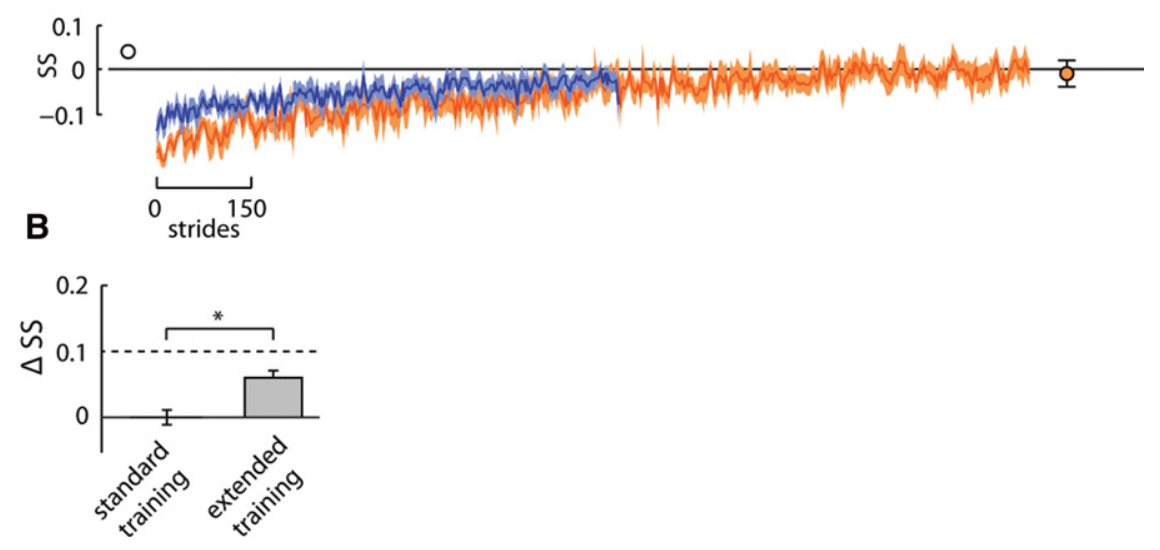

Figure 7. (A) Extended group: step symmetry (SS) across 1524 and 759 strides of the split-belt conditions on day 1 (orange) and day 2 (blue), respectively, overlaid. Solid lines with shaded area are mean \pm SE. Closed symbol shows final (last 20 strides) of symmetry for day 1 learning. Open symbol shows symmetry prior to re-learning on day 2 (last 20 strides of washout). (B) Difference across days (day 2 -day 1 ) in step symmetry during early adaptation (first 20 strides of split-belt walking) for the Extended group. 
influences retention and re-learning in children of various ages, as we did not control what the children were allowed to do between sessions in the current study.

\section{Conclusions}

Preadolescent children show clear differences in the ability to retain and re-learn adapted motor patterns. All children, regardless of age, showed retention of the adapted walking pattern; thus, they can form context specific memories. In contrast, children $<12$ yr of age did not show rapid re-learning when exposed to split-belt walking the second time. Even when given an extended period of training (i.e., reinforcement of the adapted walking pattern), young children still showed attenuated re-learning, or savings. We speculate that these young children might form a general-purpose motor memory that is not yet customized for different situations. These findings may correspond with differing rates of development of specific brain regions.

\section{Materials and Methods}

\section{Participants}

Healthy children aged 6-17 yr were recruited through flyers posted in the community and listservs of local parent and university groups. The medical history and medications of all potential participants were screened to confirm a healthy status. Ethics approval was granted by the Johns Hopkins Institutional Review Board.

\section{Experimental protocol}

To study motor adaptation, children walked on a custom-built split-belt treadmill (Woodway USA, Inc.), which has two belts, one for each leg. The experiment involved two walking conditions: (1) "tied-belt" walking, where the 2 belts move at the same speed, and (2) "split-belt" walking, where one belt (called the fast belt, randomly assigned) was made to move twice as fast as the other belt (called the slow belt). Belt speed (in meter per second) during tied-belt walking was equal to the child's leg length (in meters), measured from the greater trochanter to lateral malleolus. During split-belt walking, the slow belt speed was equal to the speed used during tied-belt walking. Belt speeds were controlled through a custom-made MATLAB computer interface (Mathworks). When walking on the treadmill, children wore a safety harness that did not support their weight and they held onto a handrail placed at chest height. Participants watched a movie while walking and were asked to avoid looking at their feet.

Children attended two testing sessions spaced $24 \mathrm{~h}$ apart. Children experienced the same experimental protocol on day 1 (Fig. 1). First, the baseline period, which consisted of 2 min of tiedbelt walking, followed by a short (10-sec) exposure to split-belt walking, and finally an additional minute of tied-belt walking. The 10-sec exposure was done to remove the "surprise" element that often increases the initial asymmetries seen with split-belt walking (Malone et al. 2011). The baseline period was followed by 15 min of split-belt walking. Short breaks were given after 5 and 10 min of split-belt walking, however, children could take breaks whenever needed. The children remained on the treadmill during breaks, either standing or sitting on a bench.

Prior to the second testing session, children were randomly allocated into either the "Retention" or "Re-learning" group (blocked randomization by age with a block size of 2). The Retention group performed 15 min of split-belt walking on day 2 , as per day 1 (Fig. 1). On day 2 children in the Re-learning group first walked for 15 min with the belts tied ("Washout", same belt speed as per day 1), followed by 15 min of split-belt walking as per day 1.

As detailed under Results, we found that children $<12$ yr old in the Re-learning group did not show faster re-learning on day 2. To determine whether increasing the exposure to the split-belt treadmill on day 1 would facilitate faster re-learning on day 2 , an additional group of children aged 6-8 yr were tested (called
"Extended" group). This age group was chosen because it was the youngest age group in this study. The Extended group experienced the same experimental protocol as the Re-learning group, with the following exceptions:

1. The duration of split-belt walking on day 1 was increased to 30 min.

2. The duration of washout (i.e., tied-belt walking) on day 2 was increased to $20 \mathrm{~min}$ to ensure that the learning from day 1 was completely unlearned.

\section{Data collection and analysis}

Kinematic data were collected at $100 \mathrm{~Hz}$ with the Optotrak system (Northern Digital, Inc.). Infrared-emitting markers were placed on the fifth metatarsal, lateral malleolus, lateral knee joint, greater trochanter, iliac crest, and acromion process bilaterally. Treadmill belt speeds, represented as voltages, were recorded directly from treadmill motor output at $1000 \mathrm{~Hz}$, and were synchronized and sampled with the Optotrak data (i.e., marker position) using Optotrak software. Data were processed using custom-made programs (Matlab, Mathworks) to calculate measures of interest.

As per previous split-belt studies, the primary measure of interest was step length symmetry, which is the normalized difference between the step lengths of the two legs (Fig. 1B; Malone and Bastian 2010; Vasudevan et al. 2011). For simplicity, the legs walking on fast and slow belts are referred to as fast and slow legs, respectively. Step length (SL) symmetry was calculated as follows:

$$
\text { SL symmetry }=\frac{\mathrm{SL}_{\text {fast }}-\mathrm{SL}_{\text {slow }}}{\mathrm{SL}_{\text {fast }}+\mathrm{SL}_{\text {slow }}}
$$

A symmetry value of 0 indicates equal step lengths. A negative value suggests that the slow SL was greater than the fast SL, and vice versa for a positive value of symmetry.

Changes in SL symmetry can result from changes in the spatial and/or temporal coordination of the legs (Malone and Bastian 2010). Therefore, we also looked at measures of spatial and temporal coordination. Our measure of spatial coordination was the center of oscillation (CO), which is the average limb angle recorded during a stride. Limb angle is the angle between the vertical and a line connecting the markers on the hip (greater trochanter) and ankle (lateral malleolus) in the sagittal plane. The center of oscillation indicates whether the leg is oscillating around a flexed (i.e., positive value), extended (i.e., negative value), or neutral (i.e., equals 0) axis. CO symmetry was calculated as follows: $\mathrm{CO}=\mathrm{CO}_{\text {fast }}-\mathrm{CO}_{\text {slow }}$

Our measure of temporal coordination was phasing of the legs. Phasing was calculated as the peak lag (as a percentage of the step cycle) of a cross-correlation function for limb angles of the two legs (slow leg was the reference leg) (Choi and Bastian 2007). Values of phasing range from 0 to 1 , with a value of 0.5 indicating symmetry (i.e., the out-of-phase or alternate coordination typically seen in walking).

SL symmetry, CO symmetry, and phasing were calculated for every stride during tied- and split-belt walking on both days. As some children walk with asymmetric gaits under tied-belt conditions (Musselman et al. 2011), the values were normalized to each child's baseline asymmetry to facilitate the comparison of averaged data across groups. To normalize, the mean baseline symmetry values for SL, CO, and phasing were calculated from the entire tied-belt walking period on day 1 , and were then subtracted from the corresponding values calculated for each stride during tiedand split-belt walking on days 1 and 2 .

We focused on two time periods during split-belt walking: (1) early adaptation (first 20 steps of split-belt walking), which reflects the initial asymmetry or error in spatial and temporal gait parameters, and (2) late adaptation (last 20 steps of split-belt walking), which reflects the symmetry value at which the adapted gait 
pattern plateaus. Mean values for the early and late adaptation periods were calculated for SL symmetry, CO symmetry and phasing on days 1 and 2 for each subject.

\section{Statistics}

Participants were divided according to experimental protocol (i.e., Retention, Re-learning, or Extended) and then further divided into four age groupings: 6-8 yr old, 9-11 yr old, $12-14 \mathrm{yr}$ old, and 15-17 yr old. To ensure that the adapted gait pattern was acquired by each child on day 1 , a one-tailed independent $t$-test comparing phasing during early and late adaptation was done for each child. If the values for step length symmetry were significantly less during the late adaptation period, then adaptation occurred. The child's data were retained for further analysis as long as adaptation was present in step length symmetry.

To assess whether baseline walking (i.e., tied-belt walking on day 1) and adaptation behavior on day 1 (i.e., mean symmetry during early and late adaptation periods) were similar between Retention and Re-learning groups as well as across age groups, a two-way ANOVA (age group $\times$ experimental group) was run for each of SL symmetry, CO symmetry and phasing at the three time points of interest (baseline walking, early adaptation, late adaptation). This comparison was also performed for the 6 to 8-yr-old Re-learning and Extended groups. Two-sample, independent $t$-tests were used to compare baseline SL symmetry, CO symmetry, and phasing between these two groups.

The Re-learning group was washed out on day 2 (i.e., they walked for $15 \mathrm{~min}$ with the belts tied prior to readaptation to splitbelt walking), and we assessed how similar the washout periods were across age groups. First, by looking at the size of the aftereffect (i.e., the first 20 steps of the washout period), we could gauge how much of the adapted pattern was still stored on day 2 . A oneway ANOVA was run on the aftereffect size across age groups for each of SL symmetry, CO symmetry, and phasing. Second, to assess whether all age groups were washed out to a similar extent, we compared the last 20 steps of the washout period (i.e., washout plateau) across age groupings with one-way ANOVA. Likewise, the washout period was compared between the 6 to 8-old Re-learning and Extended groups. Two-sample, independent $t$-tests were used to compare these two groups with respect to aftereffect size and washout plateau.

To assess the magnitude of retention or re-learning of the adapted walking pattern we compared the size of the asymmetries during early adaptation on day 1 and day 2 . For each child, the mean change in initial asymmetry across days was calculated by subtracting the mean asymmetry of day 1 from that of day 2 for each of SL symmetry, CO symmetry, and phasing. The mean differences, referred to as the early adaptation difference, for all participants in the Retention and Re-learning groups were compared using a two-way ANOVA (age group $\times$ experimental group). Pearson's correlation coefficient $(r)$ was used to further examine the relationship between age and the early adaptation difference for the Retention and Re-learning groups. Additionally, stepwise regression was used to check if age or baseline variability was a better predictor of early adaptation difference in the Re-learning group. This was done to rule out the possibility that the variability of the walking pattern in the younger children had more of an effect on the Re-learning findings than age.

To determine whether extended training on day 1 affected the magnitude of retention or re-learning on day 2 , the early adaptation difference for the 6 to 8-old Retention, Re-learning, and Extended groups were compared with a one-way ANOVA. All statistical tests were performed with Matlab (Mathworks) and the level of significance was set at 0.05. The Bonferroni correction was used for post hoc analyses. The assumption of normality was confirmed using the Shapiro-Wilk test.

\section{Competing interest statement}

The authors declare no competing financial interests.

\section{Acknowledgments}

This work was supported by a Canadian Institutes of Health Research Fellowship to K.E.M. and National Institutes of Health grants R01 HD048741 to A.J.B. and F32 NS090751 to R.T.R. We thank Katie Amenabar and Matt Statton for assistance with data collection. K.E.M.'s current affiliation is the Toronto Rehabilitation Institute-University Health Network and the Department of Physical Therapy, Faculty of Medicine, University of Toronto.

\section{References}

Adolph KE, Cole WG, Vereijken B. 2015. Intra-individual variability in the development of motor skills in childhood. In Handbook of intra-individual variability across the lifespan (ed. Diehl M, Hooker K, Sliwinski M), New York: Routledge/Taylor \& Francis Group.

Bédard P, Sanes JN. 2011. Basal ganglia-dependent processes in recalling learned visual-motor adaptations. Exp Brain Res 209: 385-393.

Caviness VS, Kennedy DN, Richelme C, Rademacher J, Filipek PA. 1996. The human brain age 7-11 years: a volumetric analysis based on magnetic resonance images. Cereb Cortex 6: 726-736.

Choi JT, Bastian AJ. 2007. Adaptation reveals independent control networks for human walking. Nat Neurosci 10: 1055-1062.

Dominici N, Ivanenko YP, Cappellini G, Zampagni ML, Lacquaniti F. 2010 Kinematic strategies in newly walking toddlers stepping over different support surfaces. J Neurophysiol 103: 1673-1684.

Forssberg H, Eliasson AC, Kinoshita H, Westling G, Johansson RS. 1995. Development of human precision grip. IV. Tactile adaptation of isometric finger forces to the frictional condition. Exp Brain Res 104: $323-330$.

Fritz SL, Rivers ED, Merlo AM, Reed AD, Mathern GD, De Bode S. 2011. Intensive mobility training postcerebral hemispherectomy: early surgery shows best functional improvements. Eur J Phys Rehabil Med 47: $569-577$.

Giedd JN, Blumenthal J, Jeffries NO, Castellanos FX, Liu H, Zijdenbos A, Paus T, Evans AC, Rapoport JL. 1999. Brain development during childhood and adolescence: a longitudinal MRI study. Nat Neurosci 2: 861-863.

Gogtay N, Giedd JN, Lusk L, Hayashi KM, Greenstein D, Vaituzis AC, Nugent TF III, Herman DH, Clasen LS, Toga AW, et al. 2004. Dynamic mapping of human cortical development during childhood through early adulthood. Proc Natl Acad Sci 101: 8174-8179.

Huang VS, Haith A, Mazzoni P, Krakauer JW. 2011. Rethinking motor learning and savings in adaptation paradigms: model-free memory for successful actions combines with internal models. Neuron 70: 787-801.

Jansen-Osmann P, Richter S, Konczak J, Kalveram KT. 2002. Force adaptation transfers to untrained workspace regions in children. Exp Brain Res 143: 212-220.

Joiner WM, Smith MA. 2008. Long-term retention explained by a model of short-term learning in the adaptive control of reaching. J Neurophysiol 100: $2948-2955$.

Kitago T, Ryan SL, Mazzoni P, Krakauer JW, Haith AM. 2013. Unlearning versus savings in visuomotor adaptation: comparing effects of washout passage of time, and removal of errors on motor memory. Front Hum Neurosci 7: 307.

Konczak J, Jansen-Osmann P, Kalveram KT. 2003. Development of force adaptation during childhood. J Mot Behav 35: 41-52.

Malone LA, Bastian AJ. 2010. Thinking about walking: effects of conscious correction versus distraction on locomotor adaptation. J Neurophysiol 103: $1954-1962$.

Malone LA, Vasudevan EVL, Bastian AJ. 2011. Motor adaptation training for faster re-learning. J Neurosci 31: 15136-15143.

Malone LA, Bastian AJ, Torres-Oviedo G. 2012. How does the motor system correct for errors in time and space during locomotor adaptation? J Neurophysiol 108: 672-683.

Marinelli L, Crupi D, Di Rocco A, Bove M, Eidelberg D, Abbruzzese G, Ghilardi MF. 2009. Learning and consolidation of visuo-motor adaptation in Parkinson's disease. Parkinsonism Relat Disord 15: 6-11.

Martin TA, Keating JG, Goodkin HP, Bastian AJ, Thach WT. 1996. Throwing while looking through prisms. II. Specificity and storage of multiple gazethrow calibrations. Brain 119: 1199-1211.

Medina JF, Garcia KS, Mauk MD. 2001. A mechanism for savings in the cerebellum. J Neurosci 21: 4081-4089.

Morton SM, Bastian AJ. 2006. Cerebellar contributions to locomotor adaptation during split-belt treadmill walking. J Neurosci 26: 9107-9116.

Musselman KE, Patrick SK, Vasudevan EVL, Bastian AJ, Yang JF. 2011. Unique characteristics of motor adaptation during walking in young children. J Neurophysiol 105: 2195-2203. 
Østby Y, Tamnes CK, Fjell AM, Westlye LT, Due-Tonnessen P, Walhovd KB. 2009. Heterogeneity in subcortical brain development: a structural magnetic resonance imaging study of brain maturation from 8 to 30 years. J Neurosci 29: 11772-11782.

Patrick SK, Musselman KE, Tajino J, Ou HC, Bastian AJ, Yang JF. 2014. Prior experience but not size of error improves motor learning on the split-belt treadmill in young children. PLoS One 9: e93349.

Reisman DS, Block HJ, Bastian AJ. 2005. Interlimb coordination during locomotion: what can be adapted and stored? J Neurophysiol 94: $2403-2415$.

Reisman DS, McLean H, Keller J, Danks KA, Bastian AJ. 2013. Repeated split-belt treadmill training improves poststroke step length asymmetry. Neurorehabil Neural Repair 27: 460-468.

Roemmich RT, Bastian AJ. 2015. Two ways to save a newly learned motor pattern. J Neurophysiol 113: 3519-3530.

Roemmich RT, Hack N, Akbar U, Hass CJ. 2014. Effects of dopaminergic therapy on locomotor adaptation and adaptive learning in persons with Parkinson's disease. Behav Brain Res 268: 31-39.

Saksena S, Husain N, Malik GK, Trivedi R, Sarma M, Rathore RS, Pandey CM, Gupta RK. 2008. Comparative evaluation of the cerebral and cerebellar white matter development in pediatric age group using quantitative diffusion tensor imaging. Cerebellum 7: 392-400.
Smith MA, Ghazizadeh A, Shadmehr R. 2006. Interacting adaptive processes with different timescales underlie short-term motor learning. PLoS Biol 4: e179.

Takahashi CD, Nemet D, Rose-Gottron CM, Larson JK, Cooper DM, Reinkensmeyer DJ. 2003. Neuromotor noise limits motor performance, but not motor adaptation, in children. J Neurophysiol 90: 703-711.

Tiemeier H, Lenroot RK, Greenstein DK, Tran L, Pierson R, Giedd JN. 2010. Cerebellum development during childhood and adolescence: a longitudinal morphometric MRI study. NeuroImage 49: 63-70.

Vasudevan EVL, Torres-Oviedo G, Morton SM, Yang JF, Bastian AJ. 2011. Younger is not always better: development of locomotor adaptation from childhood to adulthood. J Neurosci 31: 3055-3065.

Wu HG, Miyamoto YR, Gonzalez Castro LN, Olveczky BP, Smith MA. 2014. Temporal structure of motor variability is dynamically regulated and predicts motor learning ability. Nat Neurosci 17: 312-321.

Zarahn E, Weston GD, Liang J, Mazzoni P, Krakauer JW. 2008. Explaining savings for visuomotor adaptation: linear time-invariant state-space models are not sufficient. J Neurophysiol 100: 2537-2548.

Received November 6, 2015; accepted in revised form February 4, 2016. 


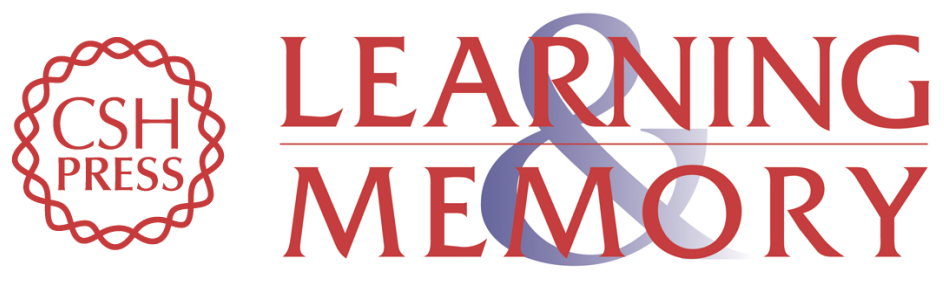

\section{Motor learning in childhood reveals distinct mechanisms for memory retention and re-learning}

Kristin E. Musselman, Ryan T. Roemmich, Ben Garrett, et al.

Learn. Mem. 2016, 23:

Access the most recent version at doi:10.1101/Im.041004.115

\begin{aligned} & \hline References $\begin{array}{l}\text { This article cites } 34 \text { articles, } 6 \text { of which can be accessed free at: } \\ \text { http://learnmem.cshlp.org/content/23/5/229.full.html\#ref-list-1 }\end{array} \\ & \begin{array}{r}\text { Creative } \\ \text { Commons } \\ \text { License }\end{array} \begin{array}{l}\text { This article is distributed exclusively by Cold Spring Harbor Laboratory Press for the } \\ \text { first } 12 \text { months after the full-issue publication date (see } \\ \text { http://learnmem.cshlp.org/site/misc/terms.xhtml). After } 12 \text { months, it is available under } \\ \text { a Creative Commons License (Attribution-NonCommercial } 4.0 \text { International), as } \\ \text { described at http://creativecommons.org/licenses/by-nc/4.0/. }\end{array} \\ & \begin{array}{c}\text { Receive free email alerts when new articles cite this article - sign up in the box at the } \\ \text { top right corner of the article or click here. }\end{array} \\ &$\hline\end{aligned}

